\title{
Effect of Sinusoidal Heating on Natural Convection Coupled to Thermal Radiation in a Square Cavity Subjected to Cross Temperature Gradients
}

\author{
Rachid El Ayachi ${ }^{1}$, Abdelghani Raji ${ }^{1}$, Mohamed Naïmi ${ }^{1}$, Hassan Elharfi ${ }^{1}$, Mohammed Hasnaoui ${ }^{2}$ \\ ${ }^{1}$ Laboratory of Flows and Transfers Modelling (LAMET), Physics Department, Faculty of Sciences and Technics, \\ Sultan Moulay Slimane University, Béni-Mellal, Morocco \\ ${ }^{2}$ Laboratory of Fluid Mechanics and Energetics (LMFE), Unit Affiliated to CNRST (URAC 27), Physics Department, \\ Faculty of Sciences Semlalia, Cadi Ayyad University, Marrakech, Morocco \\ Email: hasnaoui@ucam.ac.ma
}

Received November 30, 2012; revised January 3, 2013; accepted January 19, 2013

\begin{abstract}
Coupled natural convection and surface radiation within a square cavity, filled with air and submitted to discrete heating and cooling from all its walls, is studied numerically. The thermally active elements are centrally located on the walls of the cavity. Two heating modes, called $\boldsymbol{S B}$ and $\boldsymbol{S} \boldsymbol{V}$, are considered. They correspond to bottom and vertical left elements sinusoidally heated in time, respectively, while the top and vertical right ones are constantly cooled. The remaining portions of all the walls are considered adiabatic. The parameters governing the problem are the amplitude $(0 \leq a \leq 1)$ and the period $(0.001 \leq \tau \leq 1)$ of the temporally sinusoidal temperature, the emissivity of the walls $(0 \leq \varepsilon \leq 1)$, the relative lengths of the active elements $\left(B=h^{\prime} / H^{\prime}=\ell^{\prime} / L^{\prime}=0.5\right)$ and the Rayleigh number $\left(R a=10^{6}\right)$. The effect of such parameters on flow and thermal fields and the resulting heat transfer is examined. It is shown that, during a flow cycle, the flow structure can present complex behavior, depending on the emissivity and the amplitude and period of the exciting temperature. The rate of heat transfer is generally enhanced in the case of sinusoidal heating. Also, the resonance phenomenon existence, characterized by maximum fluctuations in flow intensity and heat transfer, is proved in this study.
\end{abstract}

Keywords: Natural Convection; Thermal Radiation; Heatlines; Cross Gradients of Temperature; Periodic Heating; Resonant Heat Transfer; Numerical Study

\section{Introduction}

Natural convection coupled with surface radiation in closed cavities has been extensively studied using numerical simulations and experiments, owing to the practical importance of such configurations in many engineering applications (convective heat losses from solar collectors, thermal design of buildings, air conditioning and, recently, electronic cooling). The majority of the existing studies, which are of numerical nature, concerned with rectangular cavities where the temperature gradient is either horizontal or vertical, including different kinds of boundary conditions [1-6]. Results of these studies show that radiation affects the dynamical and thermal structures of the fluid, reduces natural convection heat transfer component, and contributes to increase the total amount of heat exchanged in the configurations considered. Most of the works conducted in the past on natural convection coupled with radiation inside rectan- gular enclosures have been substantially oriented to study unidirectional heat transfers resulting from imposed temperature gradients (due to heat fluxes or temperature differences) either parallel or normal to gravity. In some practical situations, much more complex boundary conditions may be encountered where horizontal and vertical temperature gradients could be simultaneously imposed across the cavity. This justifies the presence of published works where the rectangular cavities are heated from below and cooled from above and simultaneously submitted to various specified thermal boundary conditions at the sidewalls. In the absence of radiation effect, Corcione [7] investigated steady natural convection of airfilled rectangular enclosures heated from below, cooled from above and submitted to various thermal boundary conditions at the sidewalls. With respect to the basic thermal configuration wherein the sidewalls are insulated, the heat transfer rate resulting from the hot bottom wall 
increases when each adiabatic sidewall is replaced with a cooled one, while showing only slight decreases as such replacement is carried out through a heated sidewall. Cianfrini et al. [8] studied natural convection heat and momentum transfer in air-filled tilted square enclosures with differentially heated opposite walls. The results obtained for the average Nusselt number of the whole cavity were expressed through a semi-empirical dimensionless correlation. More recently, Deng [9] studied laminar natural convection in a two-dimensional square enclosure having two and three discrete heat source-sink pairs on the vertical sidewalls. The effects of the size and the arrangement of the sources and sinks on the fluid flow and heat transfer characteristics were investigated. El Ayachi et al. [10] analyzed the interaction of radiation and natural convection in a square cavity filled with air and discretely heated and cooled from the four walls. In this study, the bottom active wall is at higher temperature than that of the top active wall. As results of their study, compared to pure natural convection, heat transfer across the cavity was found to be enhanced notably with the increase of the active wall emissivity, while the effect of the insulated wall emissivity was insignificant.

In the above-mentioned studies, thermal boundary conditions were assumed to be either steady isothermal or constant heat flux wall conditions. However, in many engineering applications, the energy provided to the system is variable in time and gives rise to unsteady natural-convection flow. Solar collectors and printed circuit boards are examples of such systems submitted to variable thermal boundary conditions. In addition, thermal and dynamical behaviors of a fluid subjected to time-dependent thermal conditions are impossible to predict on the basis of the results obtained with constant temperature or heat flux conditions. This justifies the presence of some works in the literature in which the variable aspect of the thermal boundary conditions was considered. In an earlier study, Lage and Bejan [11] studied theoretically the problem of natural convection in enclosures with one side heated with a pulsating heat flux. They showed that the buoyancy-induced flow resonates to a certain frequency of the pulsating heat input and the resonance phenomenon is characterized by maximum fluctuations observed in the heat transfer evolution with the period of the time-dependent temperature. The numerical results obtained by Lakhal et al. in the case of a square cavity totally [12] or partially [13] heated from below with periodic variable temperatures showed that the resulting flow structure and heat transfer were strongly dependent on the amplitude and the period of the variable temperature. Later, Lakhal et al. [14] studied transient natural convection in a square cavity isothermally cooled from above and partially heated from the side. The heating temperature was varied sinusoidally in time and in a pulsating manner. The pulsating temperature leads to better enhancement of the heat transfer in comparison with that engendered by the sinusoidal varying temperature. Antohe and Lage [15] investigated theoretically and numerically the transport of momentum and heat while considering clear fluid and fully saturated porous medium differentially heated enclosures with a time-periodic pulsating heat flux. The numerical simulations indicated that the natural-convection activity within the enclosure reaches several local maxima for certain values of the heating frequency, referred to as resonance frequency. Kwak and Hyun [16] and Kwak et al. [17] also studied such a resonant phenomenon of natural convection. It was clearly noted from their studies that the resonant frequency might be well predicted by the Brunt-Väisälä frequency, which is characterized by the thermal stratification of a system. The case of convection heat transfer was considered by Abourida et al. for a square cavity filled with air and submitted to variable thermal boundary conditions on its horizontal [18] and vertical [19] walls. Different modes of heating were considered. At high Rayleigh numbers, results reported in [18] showed that the periodic heating can be used to enhance slightly or to reduce notably the heat losses in comparison with the case of constant temperatures. For a cavity with vertical active walls [19], it was reported that the buoyancy-driven flow presents a tendency to enter in resonance with the periodic heating only when the cold temperature is maintained constant. The resonance phenomenon was not encountered when both hot and cold temperatures were varied simultaneously. Douamna et al. [20] considered two-dimensional transient natural convection in a horizontal channel periodically heated from below with a temperature varying sinusoidally with time. Results of the study showed that the three different routes leading to chaos are identified by progressively varying the amplitude and the period of the variable heating temperature. In a recent work, Zhao et al. [21] analyzed transient laminar natural convection induced by two discrete heating elements flush-mounted on one vertical wall of a square enclosure. The resonance frequencies were obtained numerically and predicted by theoretical analysis. More recently, El Ayachi et al. [22] investigated natural convection coupled with thermal radiation in a square cavity, differentially heated with a temperature varying periodically in time. The existence of the resonance phenolmenon was observed in this study and found to be more intensified by increasing the emissivity and the amplitude of the exciting temperature. The critical period of the exciting temperature leading to the resonance phenomenon was found to be independent on the amplitude the excitation and the emissivity of the walls. 
The importance of natural convection coupled with thermal radiation in a cavity subjected to periodic boundary conditions in time, is justified by the relevance of such a transitional process to many technological applications. The power supply of electronic circuits by an alternating current, the collectors of solar energy, rooms housing and building hollow blocks, in which recirculation is periodically driven by daily solar heating, are concrete examples.

In our knowledge, works dealing with time periodic combined natural convection-radiation in rectangular cavities subjected to crossed thermal gradients are nonexistent. This work is, therefore, a numerical contribution to the study of natural convection and surface radiation within a square cavity filled with air and discretely heated and cooled, in a periodic manner, from the four walls. The main parameters governing the problem are the emis-sivity of the walls and the characteristics of the exciting temperature (amplitude and period). The effect of these parameters on heat transfer and fluid flow within the cavity is examined.

\section{Problem Formulation}

The schematic view of the geometry considered in the present study is given in Figure 1. It consists of a square cavity $(A r=1)$ discretely heated and cooled from the four walls. Two heating modes are considered. In the first one, named $\boldsymbol{S B}$ heating mode, the bottom active element is heated with a temperature varying sinusoidally in time, while the vertical left active element is maintained hot at a constant temperature. In the second mode, called $\boldsymbol{S} \boldsymbol{V}$ heating mode, the vertical hot element temperature varies sinusoidally in time while that of the bottom active wall is considered constant. The inner surfaces, in contact with the fluid, are assumed to be gray, diffuse emitters and reflectors of radiation. The flow is conceived to be laminar, two-dimensional and incompressible with negligible viscous dissipation. All the thermophysical properties of the fluid are assumed constant except the density in the buoyancy term which is assumed to vary linearly with temperature (Boussinesq approximation); such a variation gives rise to the buoyancy forces.

Taking into account the above-mentioned assumptions, the non-dimensional governing equations, written in vorticity-stream function $(\Psi-\Omega)$ formulation, are as follows:

$$
\begin{aligned}
& \frac{\partial \Omega}{\partial t}+\frac{\partial(u \Omega)}{\partial x}+\frac{\partial(v \Omega)}{\partial y} \\
& =R a \operatorname{Pr} \frac{\partial T}{\partial x}+\operatorname{Pr}\left(\frac{\partial^{2} \Omega}{\partial x^{2}}+\frac{\partial^{2} \Omega}{\partial y^{2}}\right) \\
& \frac{\partial T}{\partial t}+\frac{\partial(u T)}{\partial x}+\frac{\partial(v T)}{\partial y}=\frac{\partial^{2} T}{\partial x^{2}}+\frac{\partial^{2} T}{\partial y^{2}}
\end{aligned}
$$
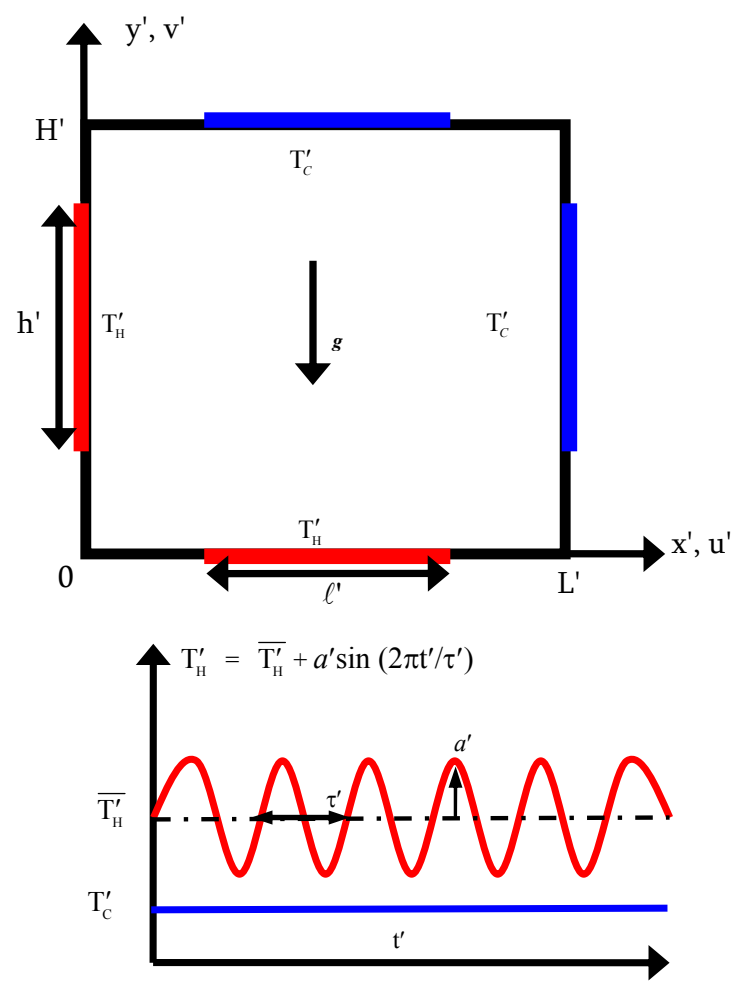

Figure 1. Studied configuration and imposed thermal excitations.

$$
\frac{\partial^{2} \Psi}{\partial x^{2}}+\frac{\partial^{2} \Psi}{\partial y^{2}}=-\Omega
$$

The dimensionless stream function and the vorticity are related to the non-dimensional velocity components by the following expressions:

$$
u=\frac{\partial \Psi}{\partial y}, v=-\frac{\partial \Psi}{\partial x} \text { and } \Omega=\frac{\partial v}{\partial x}-\frac{\partial u}{\partial y}
$$

\subsection{Boundary Conditions}

The dimensionless boundary conditions, associated to the problem are such:

$$
\begin{gathered}
u=v=\Psi=0 \text { on the cavity walls } \\
T=0 \text { on the cooled elements }
\end{gathered}
$$

$\boldsymbol{S B}$ Mode

$S \boldsymbol{V}$ Mode

$$
\left\{\begin{array}{l}
T=1+a \sin (2 \pi t / \tau) \\
\text { on the bottom heated element } \\
T=1 \\
\text { on the vertical heated element }
\end{array}\right.
$$

$$
\left\{\begin{array}{l}
T=1 \\
\text { on the bottom heated element } \\
T=1+a \sin (2 \pi t / \tau) \\
\text { on the vertical heated element }
\end{array}\right.
$$




$$
-\frac{\partial T}{\partial n}+N_{r} Q_{r}=0 \text { on the adiabatic elements }
$$

" $n$ " being the normal direction to the considered adiabatic wall.

\subsection{Coupling between Radiation and Natural Convection}

The working fluid (air) is considered to be perfectly transparent to radiation. Thus air does not participate directly to the radiative heat transfer process (the energy equation remains unchanged) but indirectly via the solid surfaces that are implicated in the radiation exchange. The coupling of the thermal model is performed by computing the radiative exchanges. The calculation of the radiative heat exchange between the internal walls of the cavity is based on the radiosity method. The grid used for convection $(81 \times 81)$ is retained in the presence of radiation and consists of 324 isothermal elementary segments. Each segment is sufficiently short to be assumed isothermal. The view factors between the isothermal elementary surfaces were determined by the Hottel's [23] crossed string method. The summation rules were checked to be sure that the view factors summation equals unity for each surface. The inner surfaces of the enclosure are assumed to be opaque, diffuse and gray. The dimensional radiosity equation for the $i^{\text {th }}$ element of the enclosure, in the case of a radiatively non-participating medium is:

$$
J_{i}^{\prime}=E_{i}+\left(1-\varepsilon_{i}\right) I_{i}^{\prime}
$$

$J_{i}^{\prime}$ is the outgoing radiation energy flux of the surface $S_{i}$. It consists of emission, $E_{i}$, plus the reflection of incoming energy, $\left(1-\varepsilon_{i}\right) I_{i}^{\prime}$.

The non-dimensional radiosity equation may be written as:

$$
J_{i}=\varepsilon_{i}\left(\frac{T_{i}}{T_{r}}+1\right)^{4}+\left(1-\varepsilon_{i}\right) \sum_{S_{j}} F_{i j} J_{j}
$$

The non-dimensional net radiative heat flux leaving a surface $S_{i}$ is evaluated by:

\subsection{Heat Transfer}

$$
Q_{r}=\varepsilon_{i}\left[\left(\frac{T_{i}}{T_{r}}+1\right)^{4}-\sum_{S_{j}} F_{i j} J_{j}\right]
$$

At each time step, the mean Nusselt numbers, characterizing the contributions of natural convection and thermal radiation through the heated walls, are evaluated as:

- on the vertical heated wall

$$
N u_{V(c v)}(t)=-\left.\int_{0.5-\frac{B}{2}}^{0.5+\frac{B}{2}}\left(\frac{\partial T}{\partial x}\right)\right|_{x=0} \mathrm{~d} y
$$

$$
N u_{V(r d)}(t)=\left.\int_{0.5-\frac{B}{2}}^{0.5+\frac{B}{2}}\left(N_{r} Q_{r}\right)\right|_{x=0} \mathrm{~d} y
$$

- on the horizontal heated wall

$$
\begin{aligned}
& N u_{H(c v)}(t)=-\left.\int_{0.5-\frac{B}{2}}^{0.5+\frac{B}{2}}\left(\frac{\partial T}{\partial y}\right)\right|_{y=0} \mathrm{~d} x \\
& N u_{H(r d)}(t)=\left.\int_{0.5-\frac{B}{2}}^{0.5+\frac{B}{2}}\left(N_{r} Q_{r}\right)\right|_{y=0} \mathrm{~d} x
\end{aligned}
$$

The instantaneous convective, radiative and total Nusselt numbers across the whole cavity are defined respectively as:

$$
\begin{aligned}
& N u_{c v}(t)=N u_{V(c v)}(t)+N u_{H(c v)}(t) \\
& N u_{r d}(t)=N u_{V(r d)}(t)+N u_{H(r d)}(t) \\
& N u(t)=N u_{c v}(t)+N u_{r d}(t)
\end{aligned}
$$

The mean Nusselt numbers, averaged in time over periods are calculated as:

$$
\begin{aligned}
\overline{N u}_{c v} & =\frac{1}{\tau_{c v}} \int_{0}^{\tau_{c v}} N u_{c v}(t) \mathrm{d} t ; \overline{N u}_{r d} \\
& =\frac{1}{\tau_{r d}} \int_{0}^{\tau_{r d}} N u_{r d}(t) \mathrm{d} t \\
\overline{N u} & =\overline{N u}_{c v}+\overline{N u}_{r d}
\end{aligned}
$$

where $\tau_{c v}$ and $\tau_{r d}$ are respectively the periods of the temporal variations of convective and radiative Nusselt numbers (they are identical in general).

\subsection{Heatlines Formulation}

Visualization of the paths followed by the heat flows through the enclosure requires the use of the heatlines concept (lines of constant heat function $H$ ). Such lines are defined through the first derivatives of the function $H$ as follows [24]:

$$
\frac{\partial H}{\partial y}=u T-\frac{\partial T}{\partial x},-\frac{\partial H}{\partial x}=v T-\frac{\partial T}{\partial y}
$$

The dimensionless heat function equation can be derived easily from Equation (15) as:

$$
\frac{\partial^{2} H}{\partial x^{2}}+\frac{\partial^{2} H}{\partial y^{2}}=-\frac{\partial(v T)}{\partial x}+\frac{\partial(u T)}{\partial y}
$$

Equation (16) is a conduction type problem with a source term becoming zero if the fluid flow subsides (fluid at rest). The solution of Equation (16) yields the 
values of the heat function in the nodes of the computational domain. The contour plots of the heat function values provide heatline patterns. The $H$ field is defined through its first-order derivatives and only differences of $H$ values are required instead of its intrinsic values. Thus, we have the freedom to state that $H(0,0)=0$ (an arbitrary reference value for the heat function $\mathrm{H}$ ). The boundary conditions for the dimensionless heat function, Equation (16), are obtained by integrating Equation (15) along the considered boundary. The following equations can be used to determine the values of heat function at the walls of the cavity:

$$
\left\{\begin{array}{l}
H(0, y)=-\int_{0}^{y} \frac{\partial T}{\partial x} \mathrm{~d} y \quad \text { for } x=0 \\
H(x, 1)=H(0,1)+\int_{0}^{x} \frac{\partial T}{\partial y} \mathrm{~d} x \text { for } y=1 \\
H(1, y)=H(1,1)-\int_{1}^{y} \frac{\partial T}{\partial x} \mathrm{~d} y \text { for } x=1 \\
H(x, 0)=H(1,0)+\int_{1}^{x} \frac{\partial T}{\partial y} \mathrm{~d} x \text { for } y=1
\end{array}\right.
$$

\section{Method of Solution}

The non linear partial differential governing equations, Equations (1)-(3), were discretized using a finite difference technique. First and second spacial derivatives were approximated by means of central differences and the time derivatives by a forward difference. The integration of Equations (1) and (2) was ensured by the Alternating Direction Implicit method (ADI). At each time step, the Poisson equation, Equation (3), was treated by using the Point Successive Over-Relaxation method (PSOR) which with an optimum over-relaxation coefficient equal to 1.92 for the grid adopted in the present study. The set of Equations (6), representing the radiative heat transfer between the different elementary surfaces of the cavity, was solved by using the Gauss-Seidel method. The numerical code was validated against the results of Akiyama and Chong [2] obtained in the case of a square cavity differentially heated. Results of comparisons, made in terms of convective Nusselt numbers, evaluated at the heated wall and presented in Table 1, show a fairly good agreement with relative maximum deviations limited to $1.07 \% /(1.36 \%)$ for $\varepsilon=0 /(1)$ for Ra varying in the range $10^{3} \leq R a \leq 10^{6}$. Results of a qualitative comparison in terms of isotherms, streamlines and heatlines, against those obtained by Deng [9] are presented in Figure 2 for $R a=10^{5}$ and $\operatorname{Pr}=0.71$ in the case of a square cavity discretely heated and cooled on the four walls without radiation effect; a good qualitative agreement can be seen by comparing these figures. Details relative to the sensitivity of the results vis-à-vis of the grid are given in Table 2. The results presented in this table justify the selected grid of $81 \times 81$ as a reasonable compromise between computational effort and required accuracy. More precisely, the maximum deviations observed in terms of $\Psi_{\min }, \Psi_{\max }, N u_{c v}$ and $N u_{r d}$ remain within $0.61 \%, 0.90 \%, 1.28 \%$ and $0.11 \%$, respectively, when the grids of $61 \times 61$ and $101 \times 101$ are considered.

\section{Results and Discussion}

The main parameters governing the problem are the amplitude of the exciting temperature $(0 \leq a \leq 1)$, it's period $(0.001 \leq \tau \leq 1)$, the emissivity of the walls

$(0 \leq \varepsilon \leq 1)$, the Prandtl number, Pr, the Rayleigh number, $\mathrm{Ra}$, and the relative length of active elements, B. To highlight the influence of $a, \tau$ and $\varepsilon$, the values of $\mathrm{B}, \operatorname{Pr}$ and $\mathrm{Ra}$ are fixed to $0.5,0.72$ (air) and $10^{6}$ respectively. In the following, the results obtained are presented in terms of temporal variations of maximum $\left(\Psi_{\max }\right)$ and minimum $\left(\Psi_{\text {min }}\right)$ stream functions and total Nusselt number $\mathrm{Nu}(\mathrm{Nu}$ being the sum of the convective and radiative Nusselt numbers). The mean values of these quantities, averaged in time over one flow cycle are also discussed to point out the effect of the variable temperature on the coupling between natural convection and surface radiation for both cases of heating considered ( $\boldsymbol{S} \boldsymbol{B}$ and $\boldsymbol{S} \boldsymbol{V}$ modes).

Table 1. Effect of $R a$ and $\varepsilon$ on $N u(c v)$, evaluated on the heating wall of a square cavity for $T_{H}^{\prime}=298.5 \mathrm{~K}$ and $T_{C}^{\prime}=2.885 \mathrm{~K}$.

\begin{tabular}{ccccc}
\hline \multicolumn{5}{c}{$\boldsymbol{\varepsilon}=\mathbf{0}$} \\
\hline Ra & $10^{3}$ & $10^{4}$ & $10^{5}$ & $10^{6}$ \\
Present work & 1.118 & 2.257 & 4.627 & 9.475 \\
$\begin{array}{c}\text { Akiyama and } \\
\text { Chong [2] }\end{array}$ & 1.125 & 2.250 & 4.625 & 9.375 \\
& & \multicolumn{2}{c}{$\boldsymbol{\varepsilon}=\mathbf{1}$} & \\
Ra & $10^{3}$ & $10^{4}$ & $10^{5}$ & $10^{6}$ \\
Present work & 1.250 & 2.242 & 4.192 & 8.100 \\
$\begin{array}{c}\text { Akiyama and } \\
\text { Chong [2] }\end{array}$ & 1.250 & 2.250 & 4.250 & 8.125 \\
\hline
\end{tabular}

Table 2. Grid sensitivity analysis for $R a=10^{6}$ and various values of $\varepsilon$.

\begin{tabular}{ccccc}
\hline \multicolumn{5}{c}{$\boldsymbol{\varepsilon = 0}$} \\
\hline Grids & $\Psi_{\min }$ & $\Psi_{\max }$ & $N u_{c v}$ & $N u_{r d}$ \\
\hline $61 \times 61$ & -32.847 & 0.0111 & 10.393 & 0 \\
$81 \times 81$ & -32.682 & 0.0112 & 10.338 & 0 \\
$101 \times 101$ & -32.622 & 0.0113 & 10.319 & 0 \\
& & $\boldsymbol{\varepsilon}=\mathbf{1}$ & & \\
Grids & $\Psi_{\min }$ & $\Psi_{\max }$ & $N u_{c v}$ & $N u_{r d}$ \\
$61 \times 61$ & -35.575 & 1.494 & 10.163 & 15.908 \\
$81 \times 81$ & -35.358 & 1.502 & 10.137 & 15.890 \\
$101 \times 101$ & -35.269 & 1.513 & 10.143 & 15.881 \\
\hline
\end{tabular}



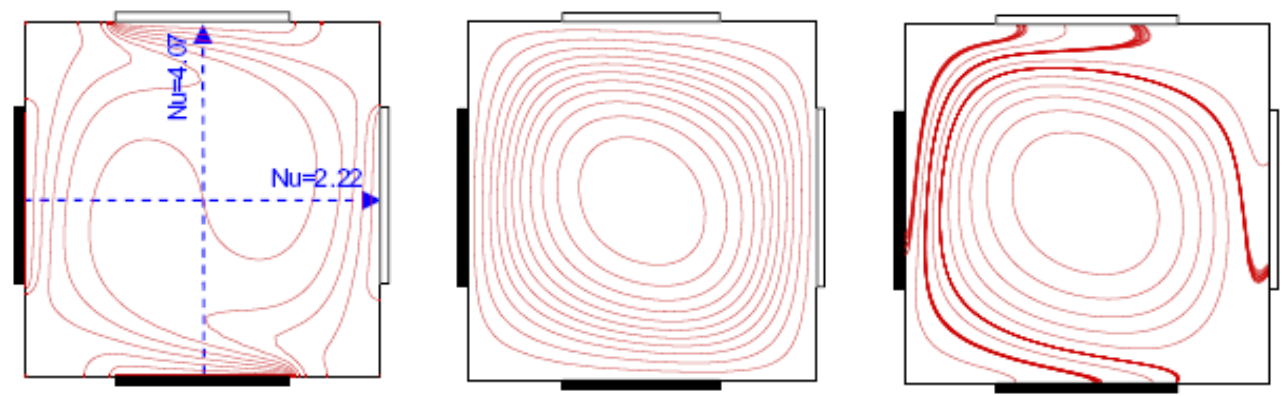

(a)
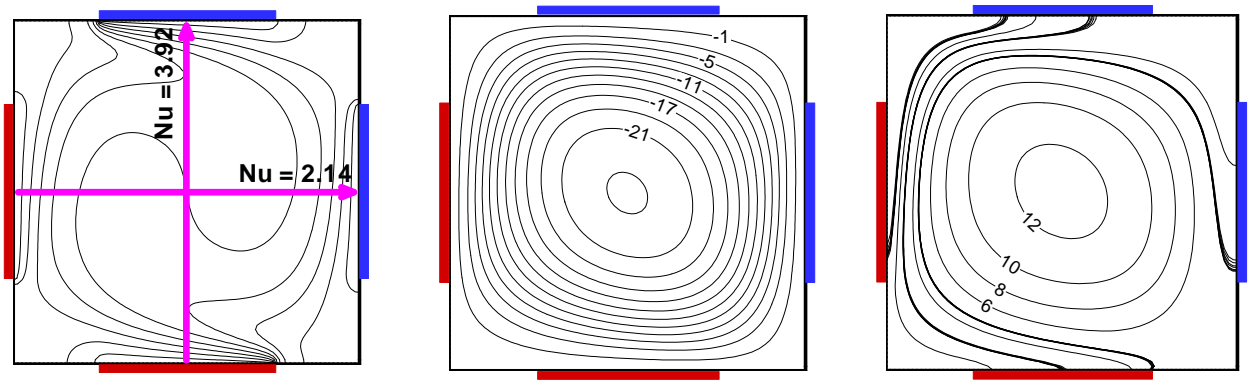

(b)

Figure 2. Numerical code validation in terms of heatlines in the case of a differentially heated square cavity for $R a=10^{5}$ and Pr = 0.7: (a) Deng [9] and (b) Our code.

\subsection{Temporal Variation of $\Psi_{\min }, \Psi_{\max }$ and $N u$}

For $\boldsymbol{S} \boldsymbol{B}$ and $\boldsymbol{S} \boldsymbol{V}$ heating modes, the evolution with time of $\Psi_{\min }, \Psi_{\max }$, and $N u$, are presented in Figures 3-5 for $\varepsilon=0$ (absence of radiation), various values of $a$, $\tau=0.008$ (for $\boldsymbol{S B}$ mode) and $\tau=0.00825$ (for $\boldsymbol{S} \boldsymbol{V}$ mode). The choice of these two values of $\tau$ is guided by a series of tests showing significant effects on fluid flow and heat transfer, which are discussed later. The reference case corresponding to a constant heating temperature $(a=0)$ is represented in these figures by a continuous horizontal line. Figures 3 and $\mathbf{4}$ show that, globally the evolution in time of $\Psi_{\min }$ and $\Psi_{\max }$ is periodic, the amplitudes of the oscillations are visibly enhanced when the amplitude $a$ of the exciting temperature is increased. It can be seen from Figure 3 that, in the case of the $\boldsymbol{S B}$ heating mode, the main flow intensity is higher in comparison to the case of $\boldsymbol{S} \boldsymbol{V}$ heating mode. It can also be noted that the monocellular flow structure, observed for $a$ $=0$, disappears in favor of a secondary flow whose size and intensity increase with $a$ (Figure 4). In terms of heat transfer, the increase of the amplitude $a$ is accompanied by a proportional increase in the amplitude of the sinusoidal evolution of $\mathrm{Nu}$ (Figure 5).

The effect of radiation on the temporal variation of $\Psi_{\min }, \Psi_{\max }$ and $N u$ is shown in Figures 6-8 in the case of highly emissive walls $(\varepsilon=1)$.

The amplitude of $\Psi_{\text {min }}$ oscillations is clearly reduced for both heating configuration considered (Figure 6),
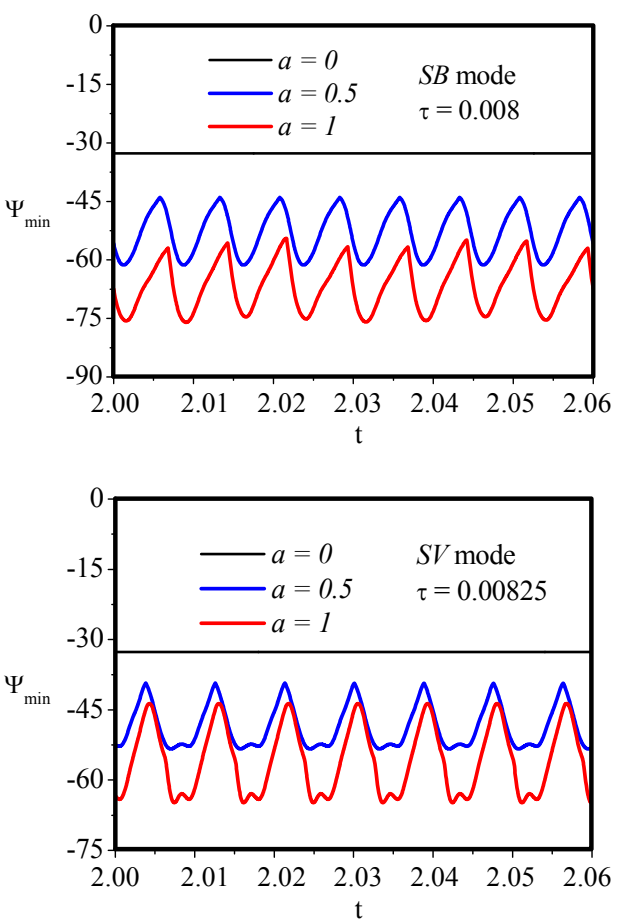

Figure 3. Effect of the amplitude $a$ on $\Psi_{\min }(t)$ for $\varepsilon=0$.

while that of $\Psi_{\max }$ is slightly affected, negatively in the case of the $\boldsymbol{S B}$ heating mode and positively in the $\boldsymbol{S} \boldsymbol{V}$ one (Figure 7). It should be noted that for the two heating modes, $\boldsymbol{S B}$ and $\boldsymbol{S} \boldsymbol{V}$, the clockwise cell, constituting the 

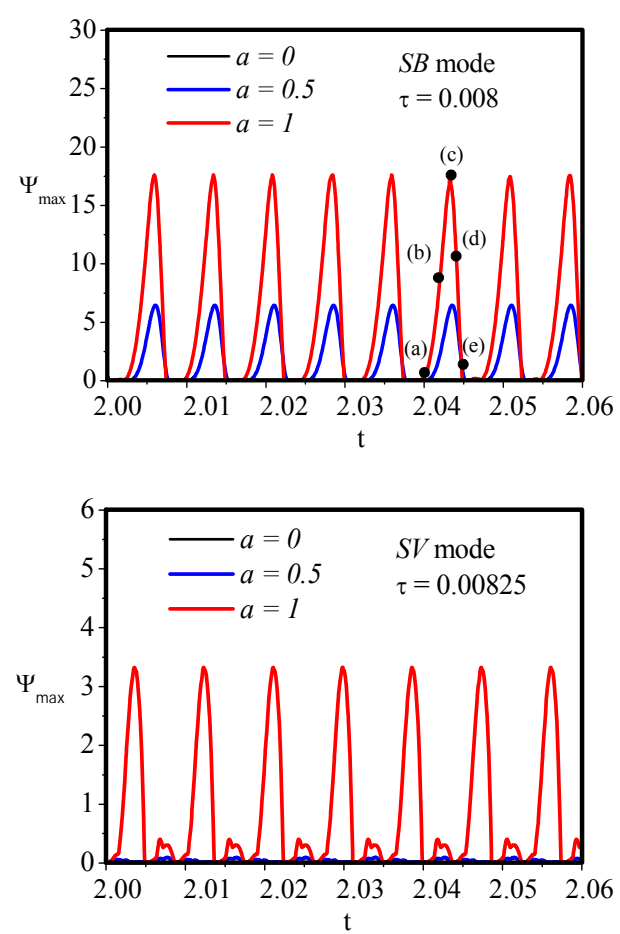

Figure 4. Effect of the amplitude $a$ on $\Psi_{\min }(t)$ for $\varepsilon=0$.
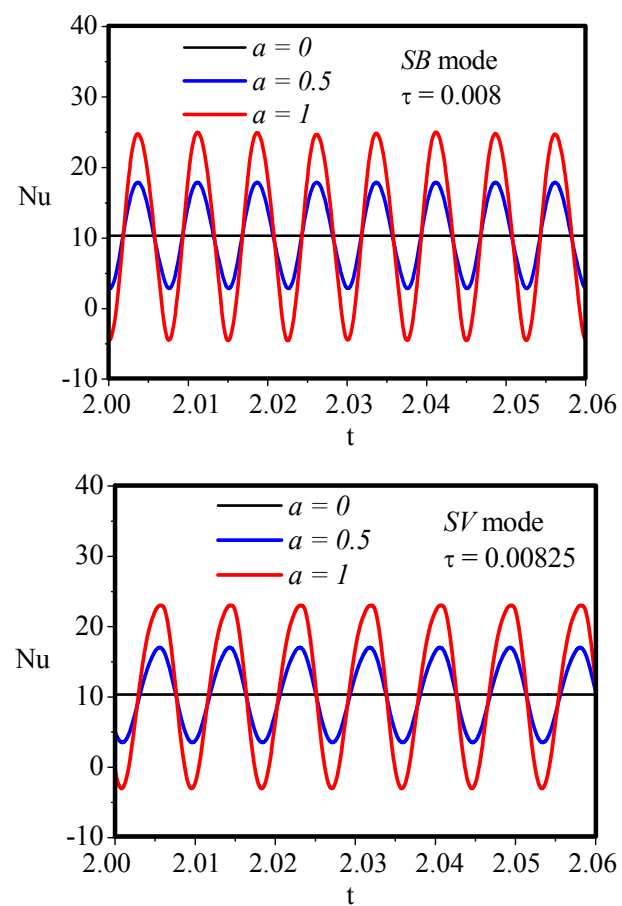

Figure 5. Effect of the amplitude $a$ on $N u(t)$ for $\varepsilon=0$.

main flow, remains dominant during the evolution of the cycle, while the secondary counterclockwise flow, plays a less important role. For the heat transfer, Figure 8, shows that when radiation of the walls is considered, the amplitude of $N u$ oscillations, is significantly enhanced; indicating an improvement of the total heat transfer when
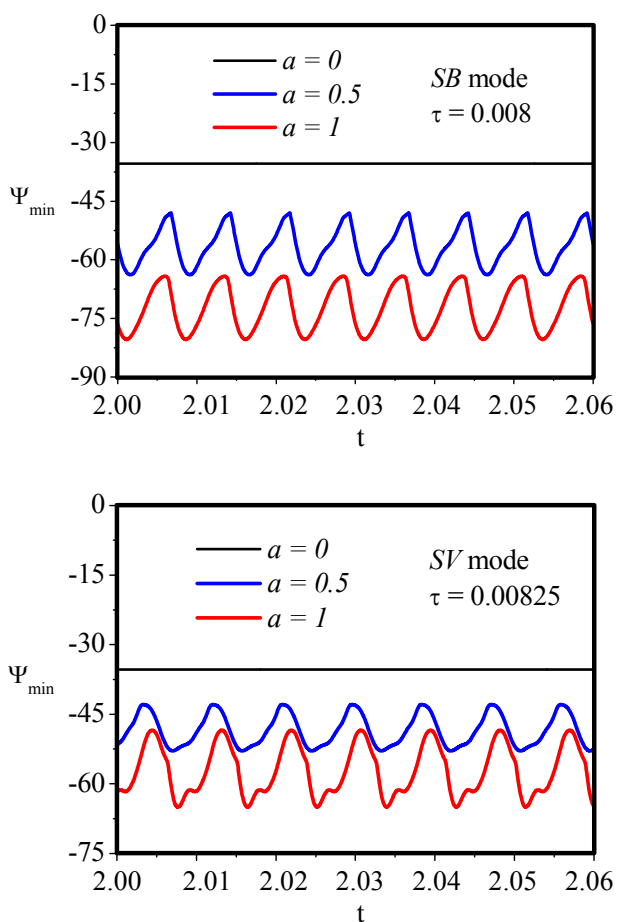

Figure 6. Effect of the amplitude $a$ on $\Psi_{\min }(t)$ for $\varepsilon=1$.
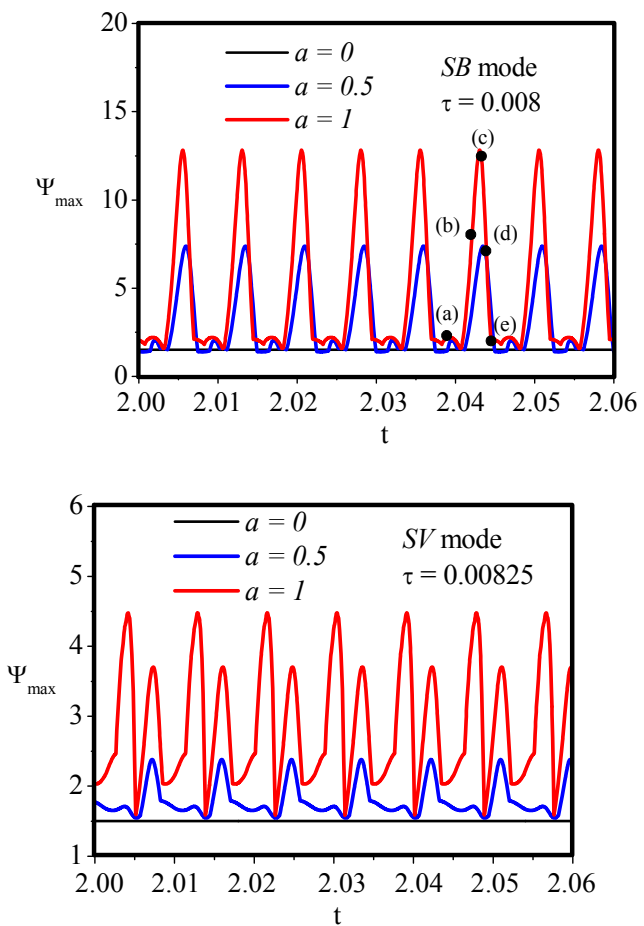

Figure 7. Effect of the amplitude $a$ on $\Psi_{\min }(t)$ for $\varepsilon=1$.

radiation is taken into account.

\subsection{Streamlines, Isotherms and Heat-Lines over a Flow Cycle}

From the temporal evolution of $\Psi_{\max }$, discussed above, it 

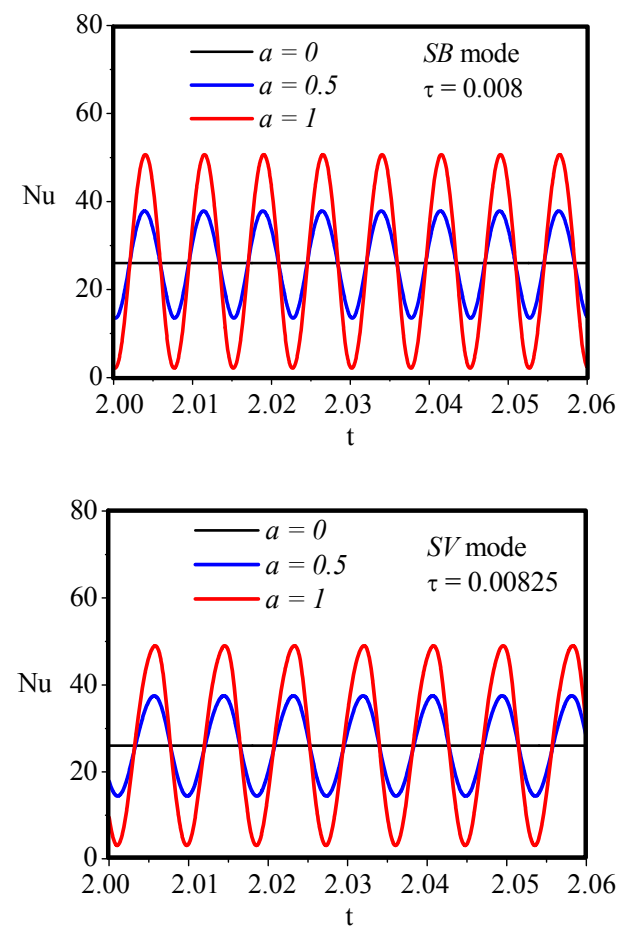

Figure 8. Effect of the amplitude $a$ on $N u(t)$ for $\varepsilon=1$. appears that the secondary flow intensity is less important in the case of $\boldsymbol{S} \boldsymbol{V}$ mode than in the $\boldsymbol{S B}$ one. That is why in this subparagraph, the study will be limited to this later configuration. Figures $\mathbf{9}$ and $\mathbf{1 0}$ correspond to the case of constant thermal excitation $(a=0)$ and are used as reference, respectively for $\varepsilon=0$ and $\varepsilon=1$.

On the other hand, in Figures $\mathbf{1 1}$ and $\mathbf{1 2}$ are presented the streamlines, isotherms and heatlines, obtained during a flow cycle in the case of sinusoidal exciting temperature $(a=1, \tau=0008)$, respectively for $\varepsilon=0$ (non-radiative walls) and $\varepsilon=1$ (strongly radiative walls). Also, in Figures 11 and 12, the letters (a), (b), (c), (d) and (e) correspond to the selected times in Figures 4 and 7. As can be seen from Figure 9, the flow is dominated mainly by a large cell zone surrounding three small others located in the central part of the cavity. Once the modulated temperature is applied $(a=1)$, a fundamental change in the flow structure is observed (Figure 11). Thus, the streamlines show that during the evolution of the cycle, the flow passes through various states characterized by the dominance, at the instant (a), of large clockwise dominant cell and the appearance, at instants (b), (c) and (d), of a secondary counterclockwise cell, small and of

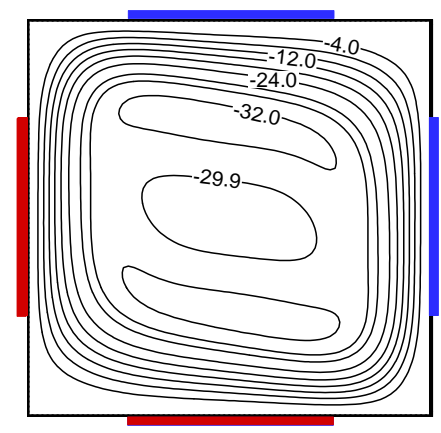

(a)

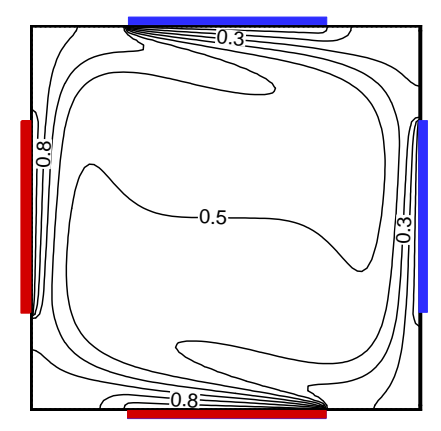

(b)

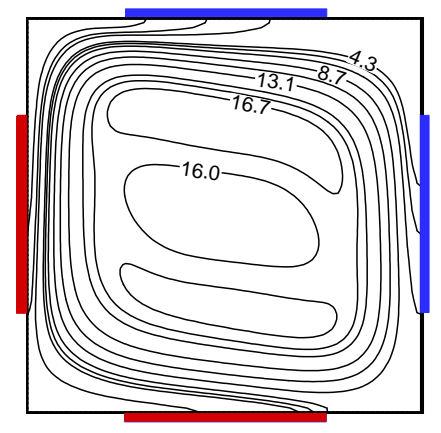

(c)

Figure 9. (a) Streamlines $\left(\Psi_{\min }=-32.68, \Psi_{\max }=0.01\right)$; (b) Isotherms and (c) Heatlines in the case of constant heating ( $\left.a=0\right)$ for $\varepsilon=0$.

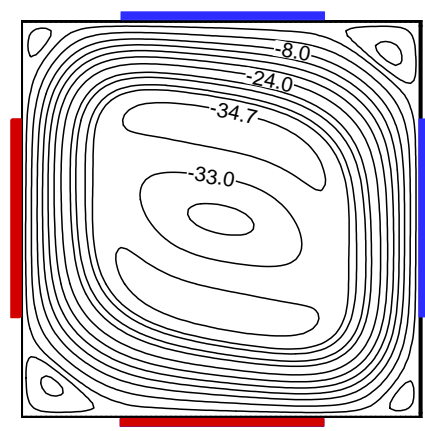

(a)

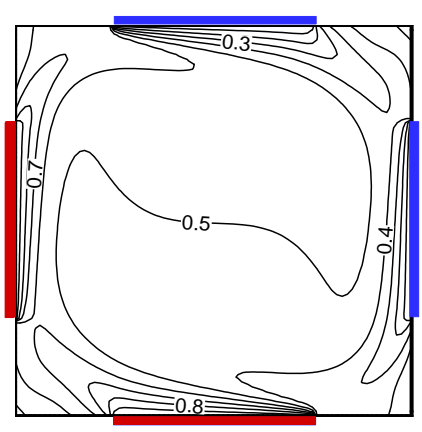

(b)

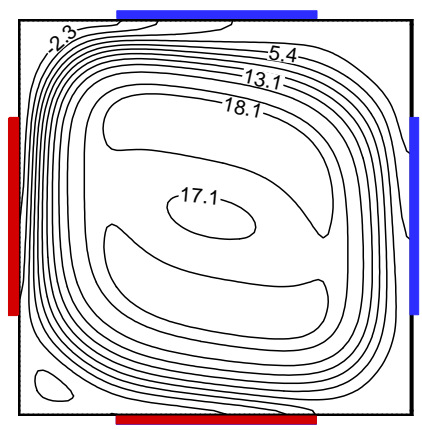

(c)

Figure 10. (a) Streamlines $\left(\Psi_{\min }=-35.36, \Psi_{\max }=1.5\right)$; (b) Isotherms and (c) Heatlines in the case of constant heating ( $\left.a=0\right)$ for $\varepsilon=1$. 

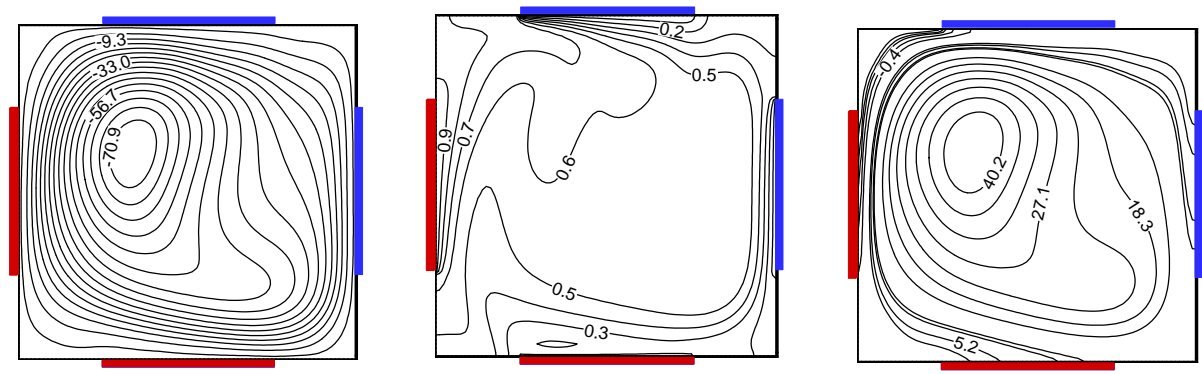

(a)
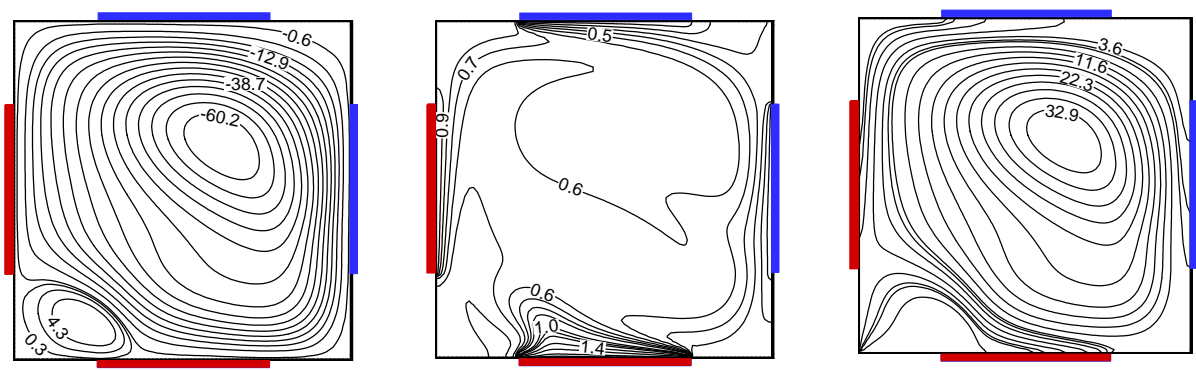

(b)
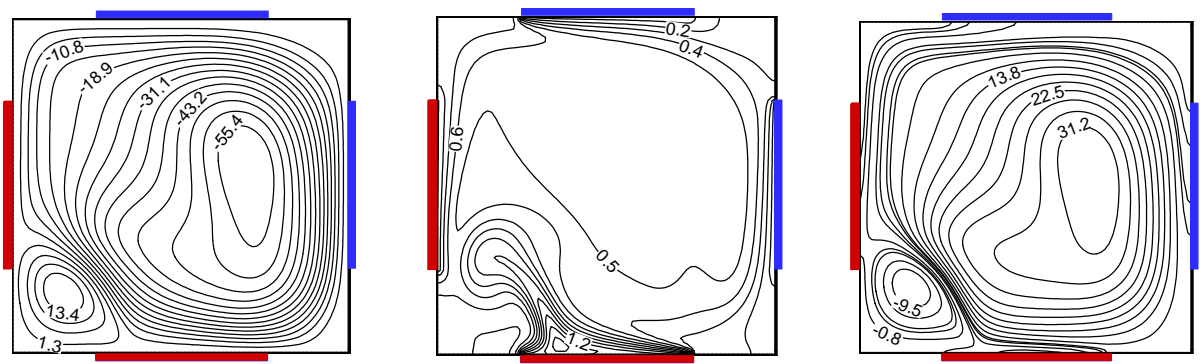

(c)
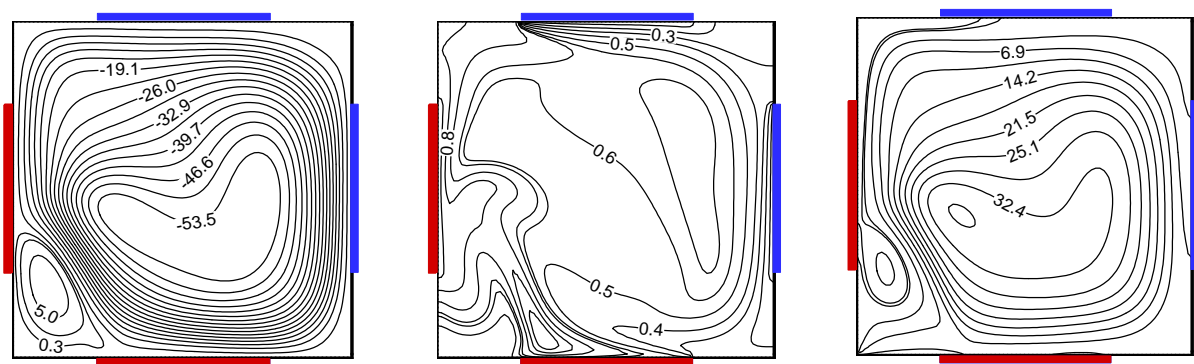

(d)
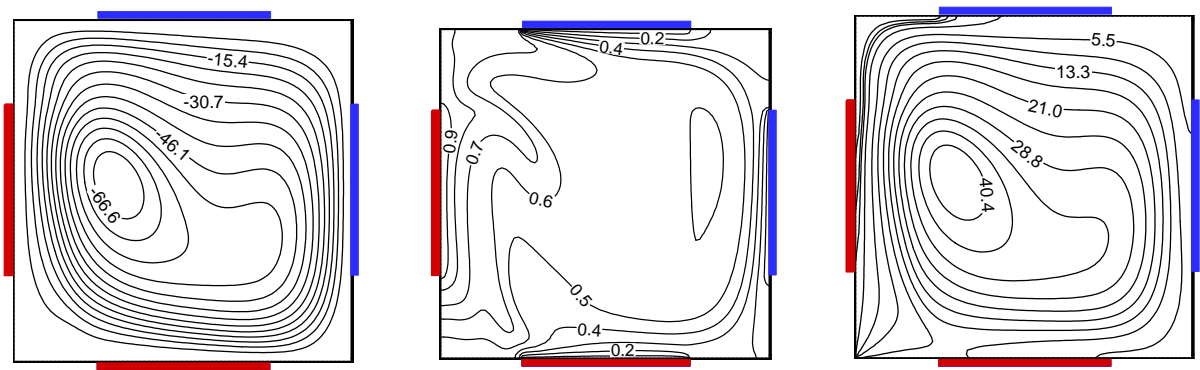

(e)

Figure 11. Streamlines (left), isotherms (center) and heatlines (right) over a flow cycle for the $S B$ mode in the case where $\varepsilon=0$. (a) $\Psi_{\min }=-75.63, \Psi_{\max }=0.124$; (b) $\Psi_{\min }=-64.52, \Psi_{\max }=8.57$; (c) $\Psi_{\min }=-59.40, \Psi_{\max }=17.49$; (d) $\Psi_{\min }=-56.9, \Psi_{\max }=8.48$; (e) $\Psi_{\min }=-71.75, \Psi_{\max }=0.03$. 

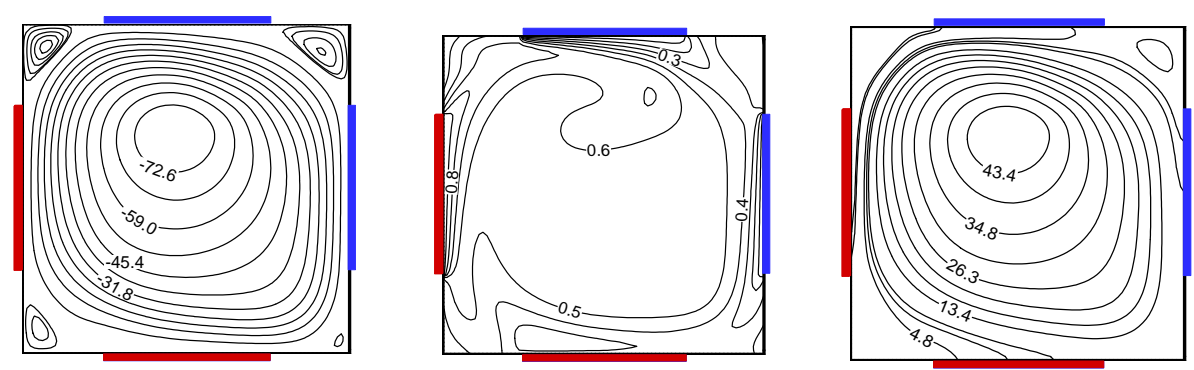

(a)
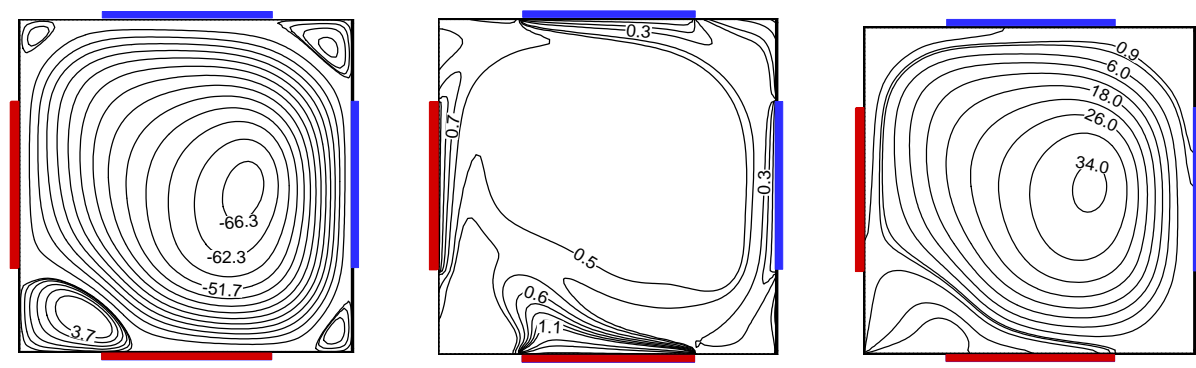

(b)
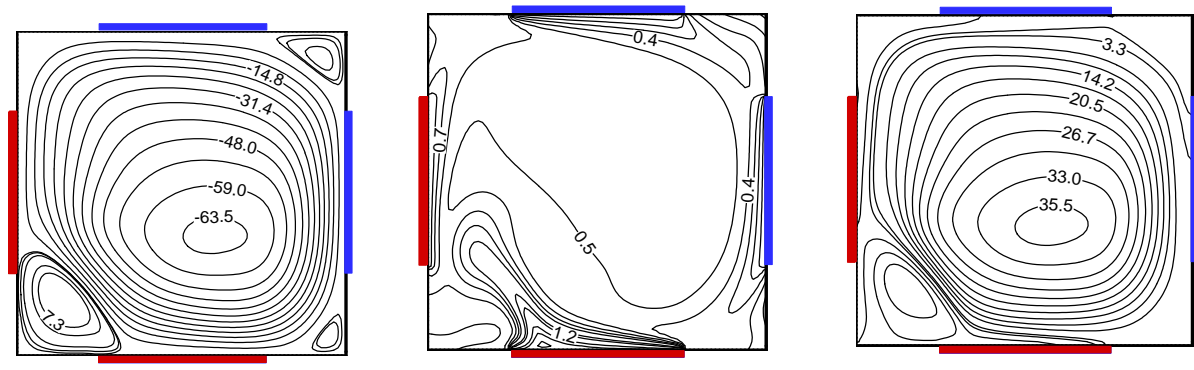

(c)
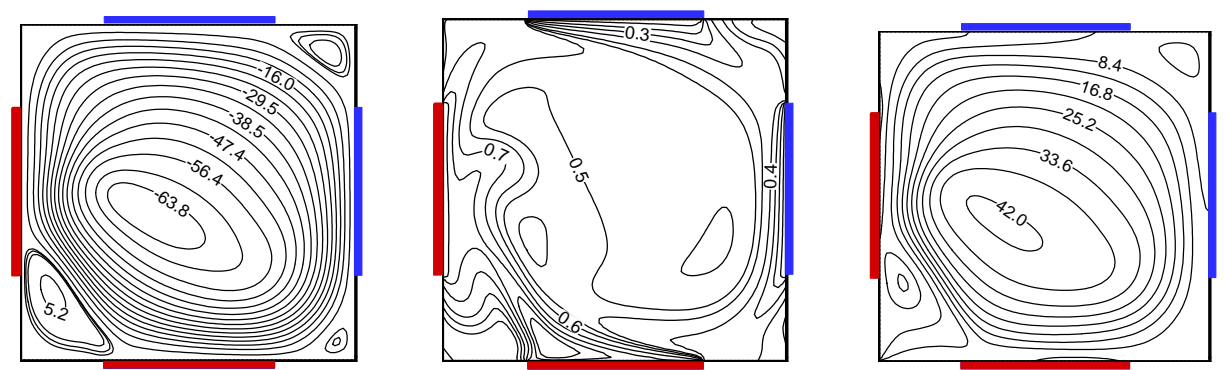

(d)
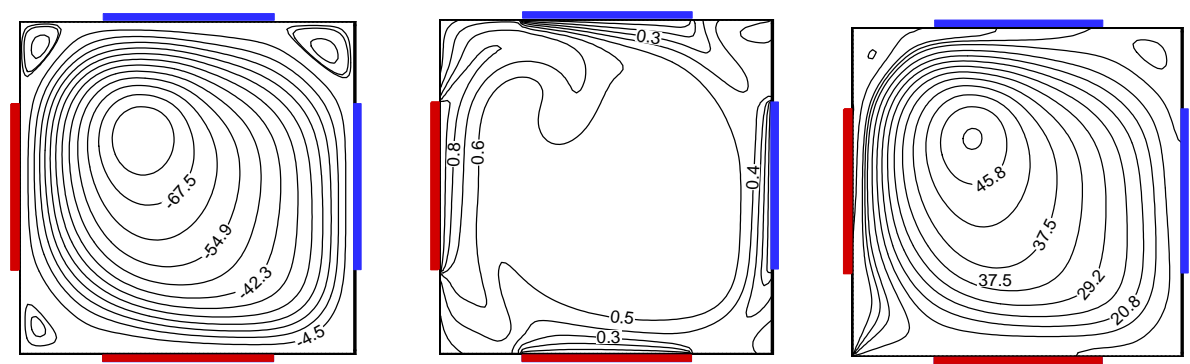

(e)

Figure 12. Streamlines (left), isotherms (center) and heatlines (right) over a flow cycle for the $S B$ mode in the case where $\varepsilon=1$. (a) $\Psi_{\min }=-79.4, \Psi_{\max }=2.2$; (b) $\Psi_{\min }=-67.63, \Psi_{\max }=6.46 ;$ (c) $\Psi_{\min }=-64.53, \Psi_{\max }=12.83 ;$ (d) $\Psi_{\min }=-65.41, \Psi_{\max }=6.43 ;($ e) $\Psi_{\min }=-80.05, \Psi_{\max }=1.83$. 
low intensity in the lower left corner of the cavity. The corresponding isotherms are tightened at the active elements, indicating that the convective heat exchange is good in this region of the cavity. Lines corresponding to heat flux (heatlines) show a similarity with the streamlines and demonstrate the dominance of convective heat transfer (high values of the stream function).

Furthermore, for $\varepsilon=1$ and $a=0$, Figure 10 shows a more complex flow structure consisting of a large and intense main cell zone and four small secondary counterclockwise cells taking place in the four corners of the cavity. By introducing a sinusoidal thermal excitation $(a=1)$, a specific examination of the streamlines of Figure 12 reveals that the flow structure, during one cycle of evolution, is dominated by a large and intense clockwise cell, surrounded, at the four corners of the cavity, by secondary counterclockwise cells more or less important according to the considered instants. Because of this complexity, the isotherms show a consistent temperature distribution, generally characterized by large thermal gradients in the vicinity of the active elements. Meanwhile, the heatlines show a similar shape to that of the streamlines, reflecting the dominance of convective heat transfer in such a situation.

\subsection{Effect of the Period $\tau$ on $\bar{\Psi}_{\max }, \bar{\Psi}_{\min }$ and $\overline{\mathrm{Nu}}$}

Based on a set of preliminary tests, the time-averaged values of the stream functions and the Nusselt number $\left(\Psi_{\max }, \Psi_{\min }\right.$, and $N u$ ), obtained for various periods, let suppose the existence of the resonance phenomenon within the cavity. This phenomenon is characterized by maximum fluctuations in the response of the system to an external excitation with well-defined period. This effect was reported in the past by several authors, both in the absence of radiation effect [11-21] and in the presence of radiation [22]. Hence, variations of $\bar{\Psi}_{\max }, \bar{\Psi}_{\text {min }}$ and $\overline{N u}$ are presented versus $\tau$ for both considered heating modes and various values of $\varepsilon$. The values corresponding to the case of the isothermal heating sources are also presented in the same figures as references.

In the absence of radiation $(\varepsilon=0)$, variations of $\bar{\Psi}_{\max }, \bar{\Psi}_{\min }$ and $\overline{N u}$ are presented in Figures 13-15, respectively.

Variations of the main flow intensity, $\bar{\Psi}_{\text {min }}$, with $\tau$ (Figure 13) show that $\left|\bar{\Psi}_{\text {min }}\right|$ increases with $\tau$ to a peak, characterizing a resonance phenomenon, for a critical value of $\tau$, which is of 0008 for the $\boldsymbol{S B}$ mode and 0.00825 for the $\boldsymbol{S} \boldsymbol{V}$ one. Such a difference between these critical periods can be attributed to the difference between the flow intensities for the two heating modes. Higher intensities of the main flow would lead to lower values of the
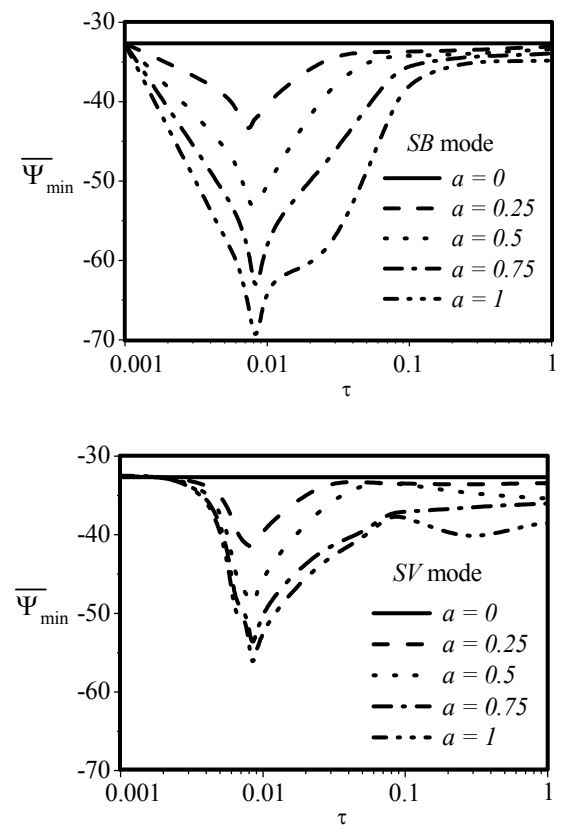

Figure 13. Variations of $\bar{\Psi}_{\min }$ with the period $\tau$ for $\varepsilon=0$ and different values of $a$.
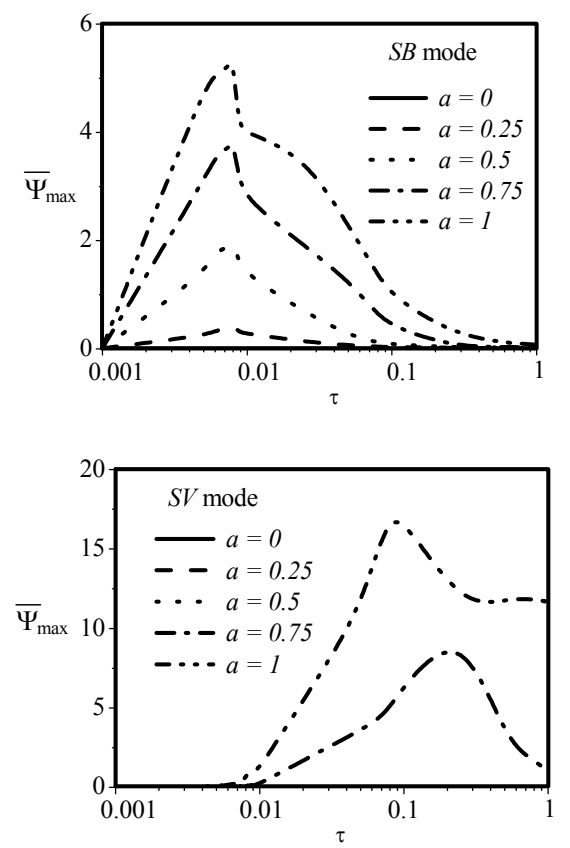

Figure 14. Variations of $\bar{\Psi}_{\max }$ with the period $\tau$ for $\varepsilon=0$ and different values of $a$.

resonant periods. Above this threshold, a further increase of $\tau$ leads to a continuous weakening of the flow intensity toward asymptotic values greater than those corresponding to the constant heating temperature $(a=0)$, reached at high values of $\tau$. By increasing the amplitude $a$ of the variable temperature, the peak becomes more important and the resonance phenomenon becomes more pronounced, 


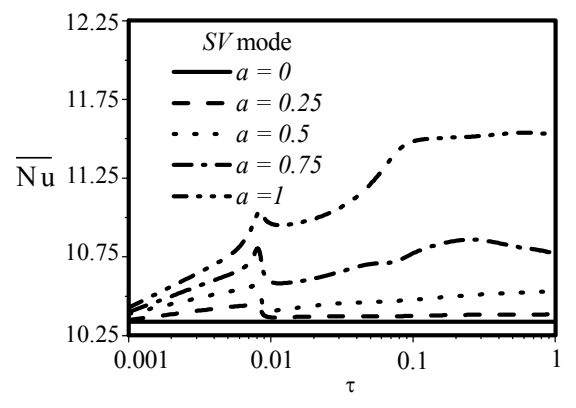

Figure 15. Variations of $\overline{N u}$ with the period $\tau$ for $\varepsilon=0$ and different values of $a$.

but without changing the critical periods.

It was reported in previous studies that the resonance occurs when the time duration, necessary for the flow to rotate through the full cavity (period of a fluid wheel [11]), is equal to that of the cyclic modulated temperature. The rotational dimensional period required for a quantity of fluid to rotate along the vertical and horizontal walls in an enclosure can be expressed as [11]:

$$
\tau^{\prime}=\frac{2\left(L^{\prime}+H^{\prime}\right)}{v^{\prime}}
$$

where $L^{\prime}$ and $H^{\prime}$ are the length and height of the enclosure, respectively. The scale of the characteristic velocity $v^{\prime}$ is determined by Lage and Bejan [11].

For the secondary flow, Figure 14 also shows a resonance phenomenon in the evolution of $\bar{\Psi}_{\max }$ which is well marked and premature for the $\boldsymbol{S B}$ mode with almost the same critical period observed in the evolution of $\bar{\Psi}_{\text {min }}$, while for the $\boldsymbol{S} \boldsymbol{V}$ mode, the occurrence of this resonance is delayed and depends on the amplitude $a$. Beyond the critical period, $\bar{\Psi}_{\max }$ decreases and stabilizes at values depending on the heating mode considered.

Regarding variations of $\overline{N u}$ with $\tau$ (Figure 15), the resonance phenomenon is also observed and appears to be more important in the case of $\boldsymbol{S B}$ mode, with critical periods identical to those on $\bar{\Psi}_{\text {min }}(0.008$ for the $\boldsymbol{S B}$ mode and 0.00825 for the $\boldsymbol{S} \boldsymbol{V}$ one). The later values are not affected by the increase of the oscillating amplitude $a$, a result in accordance with previous numerical findings [22]. Also, it is important to note that beyond the critical value of $\tau, \overline{N u}$ decreases to a minimum value reached for $\tau_{0}$ which depends on the heating mode imposed. In the case of $\boldsymbol{S B}$ mode, $\tau_{0}=0.09$, while in the case of the $\boldsymbol{S} \boldsymbol{V}$ one, $\tau_{0}$ is substantially less and is 0.01 , values beyond which $\overline{N u}$ increases until an asymptotic trend, depending on $a$.

In the case of highly emissive walls $(\varepsilon=1)$, Figures 16-18 show that the fluid circulation and the total heat transfer are more enhanced in comparison with the case of $\varepsilon=0$. When $\varepsilon$ passes from 0 to 1 , the enhancement of
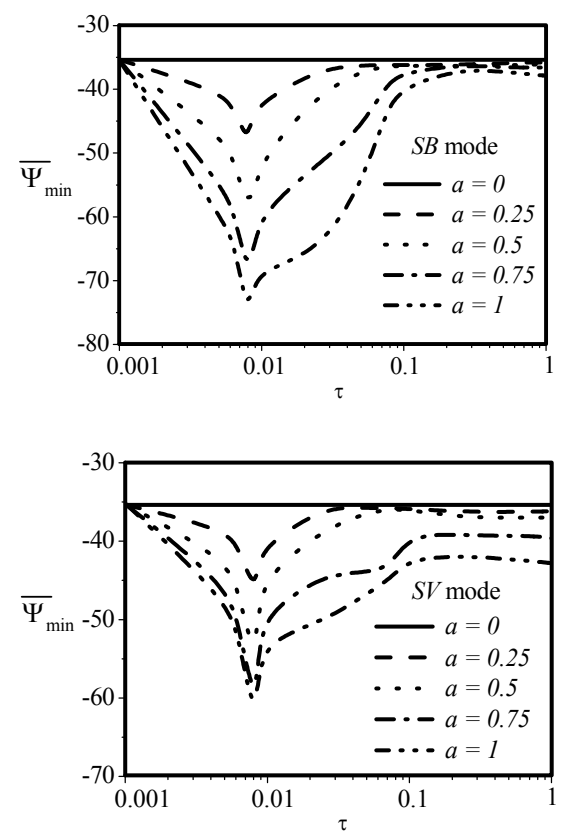

Figure 16. Variations of $\bar{\Psi}_{\min }$ with the period $\tau$ for $\varepsilon=1$ and different values of $a$.
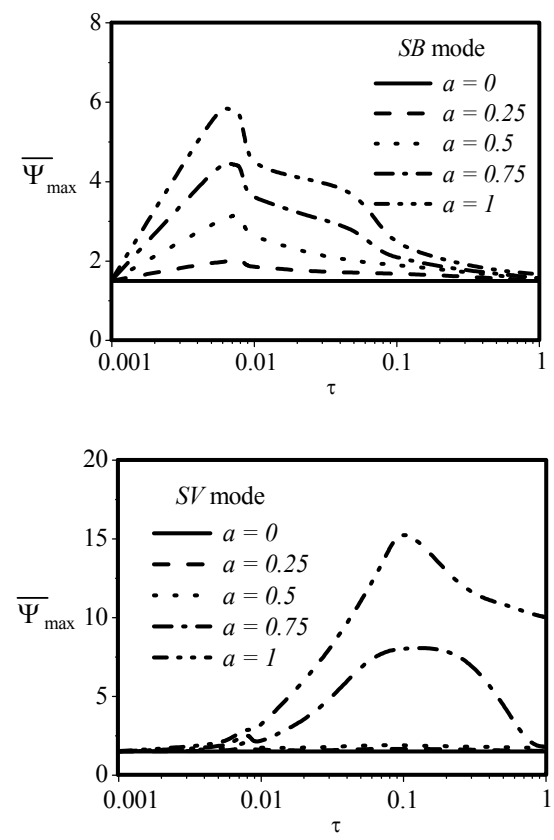

Figure 17. Variations of $\bar{\Psi}_{\max }$ with the period $\tau$ for $\varepsilon=1$ and different values of $a$.

the time averaged heat transfer, $\overline{N u}$, is of about $142 \%$ at the resonance.

The evolutions with $\tau$ are similar to those obtained without radiation effect. The resonance phenomenon is obtained for the same critical periods for both considered heating modes.

The contribution of radiation to total heat transfer 

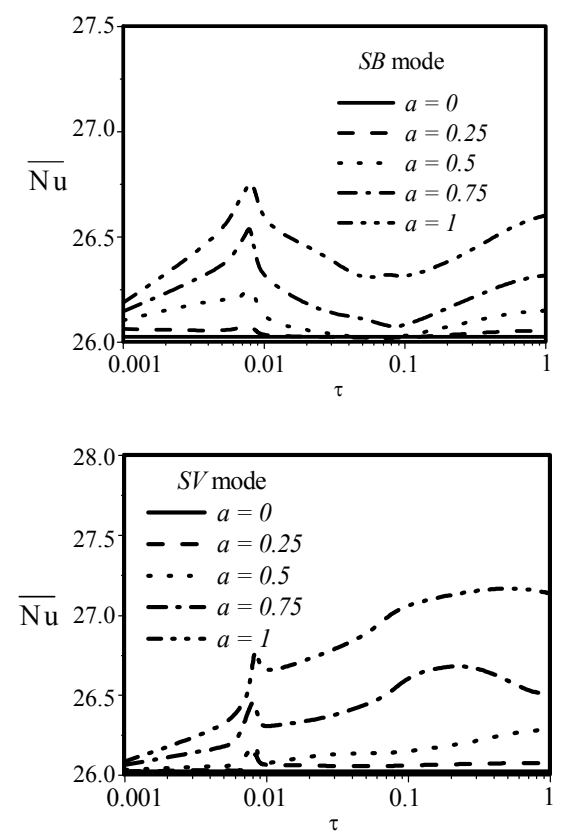

Figure 18. Variations of $\overline{N u}$ with the period $\tau$ for $\varepsilon=1$ and different values of $a$.

through the cavity is also examined in this study for the two considered configurations, $\boldsymbol{S} \boldsymbol{B}$ and $\boldsymbol{S} \boldsymbol{V}$ heating modes. The obtained results show that for a moderate value of the emissivity $(\varepsilon=0.5)$, the contribution of radiation is not negligible and it is close to that obtained for the steady state case $(a=0)$. Note that in the case of the $\boldsymbol{S V}$ mode, an increase of $a$ leads to a decrease in the contribution of radiation. In the $\boldsymbol{S} \boldsymbol{B}$ heating mode, this decrease occurs specifically for high values of $\tau$. For $\varepsilon=$ 1 , a higher contribution of radiation to total heat transfer is obtained (between 59\% and 62\%) when compared to that of natural convection.

\section{Conclusion}

The problem of natural convection coupled with thermal radiation inside a square cavity, submitted to cross gradients of temperature, is studied numerically. Two different heating modes ( $\boldsymbol{S} \boldsymbol{B}$ and $\boldsymbol{S} \boldsymbol{V})$, with a periodic variation in time, are considered. In such a situation, the solutions obtained with and without thermal radiation, are periodic in time with periods identical to that of the exciting temperature. A resonance phenomenon, characterized by maximum fluctuations of the flow intensity and heat transfer rate, is observed. The resonance period of $\boldsymbol{S B}$ mode is slightly lower than that of $\boldsymbol{S} \boldsymbol{V}$ one. Both these periods have the particularity of being independent on the excitation amplitude and the walls emissivity. Except for low values of the excitation period, time variable heating improves, generally, heat transfer than constant heating, which makes the former interesting for the heat removal through the cavity. Moreover, the control of the coupling convection-radiation, in the unsteady regime, appears to be a good means to control heat transfer for very specific values of the governing parameters.

\section{REFERENCES}

[1] C. Balaji and S. P. Venkateshan, "Interaction of Surface Radiation with Free Convection in a Square Cavity," International Journal of Heat Fluid Flow, Vol. 14, No. 3, 1993, pp. 260-267. doi:10.1016/0142-727X(93)90057-T

[2] M. Akiyama and Q. P. Chong, "Numerical Analysis of Natural Convection with Surface Radiation in a Square Cavity," Numerical Heat Transfer Part A, Vol. 31, No. 4, 1997, pp. 419-433. doi:10.1080/10407789708913899

[3] G. Colomer, M. Costa, R. Consul and A. Oliva, "Three Dimensional Numerical Simulation of Convection and Radiation in a Differentially Heated Cavity Using the Discrete Ordinates Method," International Journal of Heat Mass Transfer, Vol. 47, No. 2, 2004, pp. 257-269. doi:10.1016/S0017-9310(03)00387-9

[4] C. Y. Han and S. W. Baek, "The Effects of Radiation on Natural Convection in a Rectangular Enclosure Divided by Two Partitions," Numerical Heat Transfer Part A, Vol. 37, No. 3, 2000, pp. 249-270. doi:10.1080/104077800274280

[5] E. H. Ridouane, M. Hasnaoui, A. Amahmid and A. Raji, "Interaction between Natural Convection and Radiation in a Square Cavity Heated from Below," Numerical Heat Transfer Part A, Vol. 45, No. 3, 2004, pp. 289-311. doi:10.1080/10407780490250373

[6] R. El Ayachi, A. Raji, M. Hasnaoui and A. Bahlaoui, "Combined Effect of Radiation and Natural Convection in a Square Cavity Differentially Heated with a Periodic Temperature," Numerical Heat Transfer Part A, Vol. 53, No. 12, 2008, pp. 1339-1356. doi: $10.1080 / 10407780801960043$

[7] M. Corcione, "Effects of the Thermal Boundary Conditions at the Sidewalls upon Natural Convection in Rectangular Enclosures Heated from Below and Cooled from Above," International Journal of Thermal Sciences, Vol. 42, No. 2, 2003, pp. 199-208. doi:10.1016/S1290-0729(02)00019-4

[8] C. Cianfrini, M. Corcione and P. P. Dell'Omo, "Natural Convection in Tilted Square Cavities with Differentially Heated Opposite Walls," International Journal of Thermal Sciences, Vol. 44, No. 5, 2005, pp. 441-451. doi:10.1016/j.ijthermalsci.2004.11.007

[9] Q.-H. Deng, "Fluid Flow and Heat Transfer Characteristics of Natural Convection in Square Cavities Due to Discrete Source-Sink Pairs," International Journal of Heat Mass Transfer, Vol. 51, No. 25-26, 2008, pp. 5949-5957. doi:10.1016/j.ijheatmasstransfer.2008.04.062

[10] R. El Ayachi, A. Raji, M. Hasnaoui, A. Abdelbaki and M. Naïmi, "Combined Effects of Radiation and Natural Convection in a Square Cavity Submitted to Cross Gradients of Temperature: Case of Partial Heating and Cooling," Computational Thermal Sciences, Vol. 3, No. 1, 2011, pp. 
73-78. doi:10.1615/ComputThermalScien.v3.i1.60

[11] J. L. Lage and A. Bejan, "The Resonance of Natural Convection in a Horizontal Enclosure Heated Periodically from the Side," International Journal of Heat Mass Transfer, Vol. 36, No. 8, 1993, pp. 2027-2038. doi:10.1016/S0017-9310(05)80134-6

[12] E. K. Lakhal, M. Hasnaoui, P. Vasseur and E. Bilgen, "Convection Naturelle Dans une Cellule Carrée Chauffée Périodiquement par le Bas: Étude Numérique," Revue Generale de Thermique, Vol. 392, No. 392-393, 1994, pp. 480-485.

[13] E. K. Lakhal, M. Hasnaoui, P. Vasseur and E. Bilgen, "Natural Convection in a Square Enclosure Heated Periodically from Part of the Bottom Wall," Numerical Heat Transfer Part A, Vol. 27, No. 3, 1995, pp. 319-333. doi:10.1080/10407789508913703

[14] E. K. Lakhal, M. Hasnaoui and P. Vasseur, "Numerical Study of Transient Natural Convection in a Cavity Heated Periodically with Different Types of Excitations," International Journal of Heat Mass Transfer, Vol. 42, No. 21, 1999, pp. 3927-3941. doi:10.1016/S0017-9310(99)00070-8

[15] B. V. Antohe and J. L. Lage, "Amplitude Effect on Convection Induced by Time-Periodic Horizontal Heating," International Journal of Heat Mass Transfer, Vol. 39, No. 6, 1996, pp. 1121-1133. doi:10.1016/0017-9310(95)00207-3

[16] H. S. Kwak and J. M. Hyun, "Natural Convection in an Enclosure Having a Vertical Sidewall with Time Varying Temperature," Journal of Fluid Mechanics, Vol. 329, 1996, pp. 65-88. doi:10.1017/S0022112096008828

[17] H. S. Kwak, K. Kuwahara and J. M. Hyun, "Resonant Enhancement of Natural Convection Heat Transfer in a Square Enclosure," International Journal of Heat Mass
Transfer, Vol. 41, No. 18, 1998, pp. 2837-2846. doi:10.1016/S0017-9310(98)00018-0

[18] B. Abourida, M. Hasnaoui and S. Douamna, "Transient Natural Convection in a Square Enclosure with Horizontal Walls Submitted to Periodic Temperatures," Numerical Heat Transfer Part A, Vol. 36, No. 7, 1999, pp. 737-750. doi:10.1080/104077899274543

[19] B. Abourida, M. Hasnaoui and S. Douamna, "Convection Naturelle Dans une Cavité Carrée Avec des Parois Verticales Soumises à des Températures Périodiques," Revue Generale de Thermique, Vol. 37, No. 9, 1998, pp. 788800.

[20] S. Douamna, M. Hasnaoui and B. Abourida, "Two-Dimensional Transient Natural Convection in a Repetitive Geometry Submitted to Variable Heating from Below: Numerical Identification of Routes Leading to Chaos," $\mathrm{Nu}$ merical Heat Transfer Part A, Vol. 37, No. 7, 2000, pp. 779-799. doi:10.1080/104077800274073

[21] F. Y. Zhao, D. Liu and G. F. Tang, "Resonant Response of Fluid Flow Subjected to Discrete Heating Elements," Energy Conversion Management, Vol. 48, No. 9, 2007, pp. 2461-2472. doi:10.1016/j.enconman.2007.04.008

[22] R. El Ayachi, A. Raji, M. Hasnaoui and A. Bahlaoui, "Combined Effect of Radiation and Natural Convection in a Square Cavity Differentially Heated with a Periodic Temperature," Numerical Heat Transfer Part A, Vol. 53, No. 12, 2008, pp. 1339-1356. doi: $10.1080 / 10407780801960043$

[23] H. C. Hottel and A. F. Saroffim, "Radiative Heat Transfer," McGraw-Hill, New York, 1967.

[24] S. Kimura and A. Bejan, "The Heatline Visualization of Convective Heat Transfer," ASME Journal of Heat Transfer, Vol. 105, No. 4, 1983, pp. 916-919. doi:10.1115/1.3245684 


\section{Nomenclature}

$A_{r}$ : aspect ratio of the cavity $\left(A_{r}=L^{\prime} / H^{\prime}=1\right)$

$B$ : relative lengths of the active portions,

$B=h^{\prime} / H^{\prime}=\ell^{\prime} / L^{\prime}$

$c v$ : convection

$F_{i j}$ : view factor from $S_{i}$ surface to $S_{j}$ one

$g$ : acceleration due to gravity, $\mathrm{m} / \mathrm{s}^{2}$

$h^{\prime}$ : length of the vertical active portions, $\mathrm{m}$

$H^{\prime}$ : height of the cavity, $\mathrm{m}$

$J_{i}$ : dimensionless radiosity, $J_{i}^{\prime} / \sigma T_{C}^{\prime 4}$

$\ell^{\prime}$ : width of the horizontal active elements, $m$

$L^{\prime}$ : length of the cavity, $\mathrm{m}$

$N_{r}$ : convection-radiation interaction parameter,

$N_{r}=\sigma T_{C}^{\prime 4} H^{\prime} / \lambda\left(T_{H}^{\prime}-T_{C}^{\prime}\right)$

$N u$ : average Nusselt number

Pr: Prandtl number, $\operatorname{Pr}=v / \alpha$

$Q_{r}$ : dimensionless radiative heat flux, $Q_{r}=Q_{r}^{\prime} / \sigma T_{C}^{\prime 4}$

$R a$ : Rayleigh number, $R a=g \beta\left(T_{H}^{\prime}-T_{C}^{\prime}\right) H^{\prime 3} / \alpha v$

$r d$ : radiation

$t$ : dimensionless time, $t=t^{\prime} \alpha / H^{\prime 2}$

$T^{\prime}$ : dimensional fluid temperature, $\mathrm{K}$

$T$ : dimensionless fluid temperature,

$T=\left(T^{\prime}-T_{C}^{\prime}\right) /\left(T_{H}^{\prime}-T_{C}^{\prime}\right)$

$T_{C}^{\prime}$ : temperature of the cold elements, $\mathrm{K}$

$T_{H}^{\prime}$ : temperature of the heated elements, $\mathrm{K}$

$\Delta T^{\prime}$ : temperature difference, $\Delta T^{\prime}=T_{H}^{\prime}-T_{C}^{\prime}$

$T_{r}$ : dimensionless reference temperature,
$T_{r}=T_{C}^{\prime} /\left(T_{H}^{\prime}-T_{C}^{\prime}\right)$

$(u, v)$ : dimensionless horizontal and vertical velocities,

$(u, v)=\left(u^{\prime}, v^{\prime}\right) H^{\prime} / \alpha$

$(x, y)$ : dimensionless coordinates, $(x, y)=\left(x^{\prime}, y^{\prime}\right) / H^{\prime}$

\section{Greek Symbols}

$\alpha:$ thermal diffusivity of fluid, $\mathrm{m}^{2} / \mathrm{s}$

$\beta$ : thermal expansion coefficient of fluid, $1 / \mathrm{K}$

$\varepsilon:$ emissivity of the walls

$\lambda:$ thermal conductivity of fluid, $\mathrm{W} /(\mathrm{K} \times \mathrm{m})$

$v$. kinematic viscosity of fluid, $\mathrm{m}^{2} / \mathrm{s}$

$\tau$. dimensionless period of the heating temperature,

$\tau=\tau^{\prime} \alpha / H^{\prime 2}$

$\Omega$ : dimensionless vorticity, $\Omega=\Omega^{\prime} H^{\prime 2} / \alpha$

$\Psi$ : dimensionless stream function, $\Psi=\Psi^{\prime} / \alpha$

$\sigma:$ Stéfan-Boltzman constant,

$\sigma=5.6697 \times 10^{-8} \mathrm{~W} /\left(\mathrm{m}^{2} \cdot \mathrm{K}^{4}\right)$

\section{Subscripts}

$C$ : cooled surface

$H$ : heated surface

\section{Superscripts}

': dimensional variable 